\title{
PROGRAMA DE PRIMEIRA AJUDA EM SAÚDE MENTAL EM ESTUDANTES DO 1. ANO ENSINO SUPERIOR
}

\author{
Sandrina Ribeiro da Cunha \\ Escola Superior de Enfermagem de Coimbra \\ sandrinacunha@esenfc.pt \\ Luís Manuel de Jesus Loureiro \\ Escola Superior de Enfermagem de Coimbra \\ Unidade de Investigação em Ciências da Saúde: Enfermagem \\ luisloureiro@esenfc.pt
}

Recepción Artículo: 16 mayo 2021

Admisión Evaluación: 16 mayo 2021

Informe Evaluador 1: 21 mayo 2021

Informe Evaluador 2: 26 mayo 2021

Aprobación Publicación: 02 junio 2021

\section{RESUMO}

Antecedentes: 0 programa Primeiros Socorros em Saúde Mental (PASM) é uma ferramenta educacional utilizada no contexto da promoção da literacia em saúde mental nos mais diversos contextos, e cuja evidência científica é apresentada como eficaz. Objectivo: analisar a eficácia do programa PASM nos alunos do $1^{\circ}$ ano do curso de Enfermagem. Metodologia: concepção pré-experimental, com um único grupo, avaliação pré e pós intervenção, aplicando uma amostra de 188 alunos do $1^{0}$ ano do curso de Enfermagem. 0 instrumento de recolha de dados utilizado foi 0 QuALiSMental. Os dados foram analisados utilizando o software IBM SPSS Statistics v24.0. Resultados: o programa é eficaz em termos de reconhecimento da ansiedade social, intenção de pedir ajuda e confiança para prestar ajuda. Conclusão: 0 programa PSSM revela-se adequado no contexto da integração dos estudantes no ensino superior, e pode ser utilizado como medida preventiva e para promover a saúde mental.

Palavras-chave: literacia em saúde mental; primeiros socorros; jovens; enfermagem; ansiedade social.

\section{ABSTRACT}

First aid program in mental health in 1st year college students. Background: The Mental Health First Aid (MHFA) program is an educational tool used in the context of promoting literacy in mental health in the most diverse contexts, and whose scientific evidence is presented as effective. Objective: to analyze the effectiveness of the MHFA program on 1st year students of the Nursing 
course. Methodology: Pre-experimental design, with a single group, pre- and post-intervention assessment, applying a sample of 1881 st year students of the Nursing course. The data collection instrument used was the QuALiSMental. The data were analyzed using the IBM SPSS Statistics v24.0 software.

Results: the program is effective in terms of recognizing social anxiety, intention to request help and confidence in providing help. Conclusion: The MHFA program proves to be adequate in the context of the integration of students in higher education, and can be used as a preventive measure and promoting mental health

Keywords: mental health literacy; first aid; young; nursing; social anxiety

\section{INTRODUÇÃO}

A literacia em saúde mental (LSM) é hoje perspetivada como um conceito fundamental para quem procura desenvolver intervenções em contextos comunitários promovendo, quer o conhecimento em saúde mental, quer ainda capacitando os cidadãos para agirem em prol da sua saúde mental e daqueles que os rodeiam (Jorm, 2019; Loureiro \& Freitas, 2020a). Se nortearmos estas intervenções pela definição do conceito de literacia em saúde mental como proposto por Jorm (2019), as ações planeadas e propostas devem apontar para um incremento, quer da capacidade de reconhecer os problemas de saúde mental (perturbações incluídas) de modo a prestar ajuda e apoio, quer ainda a sua gestão no quotidiano. A prestação de primeira ajuda em saúde mental como sabemos, decorre da operacionalização do conceito de literacia em saúde mental e é o corolário do movimento iniciado na Austrália por Jorm (Jorm et al., 1997) em finais dos anos noventa do século XX.

Neste sentido o programa de primeira ajuda em saúde mental, nome definido no contexto português por Loureiro (2014), procura promover a LSM no sentido de aumentar os conhecimentos em todas as componentes da literacia em saúde mental e simultaneamente capacitar as pessoas para agirem em prol da sua saúde e daqueles que os rodeiam no quotidiano (Loureiro et al, 2020).

No caso dos estudantes do ensino superior esta preocupação torna-se premente, pois a evidência mostra que existe uma elevada prevalência de problemas de saúde mental e perturbações nestes jovens, com enfase para os problemas relacionados a ansiedade e as perturbações de humor como a depressão, alguns pré-existentes e que podem agravar-se, e outros que tendem a surgir à medida que o percurso académico avança (Cunha, 2019; Loureiro, 2020), comprometendo todas as vertentes do sucesso académico.

Nos estudantes que frequentam cursos de saúde como são os de enfermagem, pela sua natureza e especificidade, estes problemas tendem a destacar-se como uma principais ameaças à saúde e bem-estar dos estudantes e consequentemente ao seu futuro pessoal e profissional (Loureiro \& Freitas, 2020a; 2020b)

0 programa de primeira ajuda em saúde mental apresenta-se como umas das ferramentas que permite e pode contribuir para que as instituições intervenham no sentido de gerirem e minimizarem o impacto destes problemas de modo a que nas situações de ameaça ao bem-estar e em situações de adoecer mental dos estudantes, a primeira ajuda promova o reconhecimento precoce dos problemas e favoreça a procura de ajuda especializada de modo a que estes recuperem a boa saúde mental (Loureiro, 2020). Simultaneamente pode reduzir o estigma social associado a estes problemas de saúde, estigma esse que se constitui como um forte inibidor à procura de ajuda e que tende a aumentar o sofrimento pessoal.

Essa ajuda por ser prestada interpares, quer ainda por docentes e outros profissionais das instituições nas relações que estabelecem no quotidiano com os estudantes em que se observam eventuais mudanças no padrão comportamental que possam indiciar um problema de saúde mental (Cunha, 2020). 


\section{OBJETIVO DA INVESTIGAÇÃO}

0 presente estudo tem como objetivo avaliar a efetividade do programa de PASM na literacia acerca da ansiedade social dos estudantes do 1. ano da licenciatura em Enfermagem de uma instituição da região centro de Portugal continental em termos do reconhecimento da ansiedade, intenção de procura de ajuda e confiança para prestar ajuda.

\section{AMOSTRA}

A amostra é constituída por 188 estudantes com uma média de idades de

18,61 anos (desvio padrão de 1,97 anos) que frequentam 0 1. ano do CLE de uma instituição de ensino superior da Região Centro de Portugal Continental. Em termos de características e como se pode observar da Tabela 1, dos 188 estudantes, $14,90 \%$ são do sexo masculino e $85,10 \%$ do feminino. Em termos de residência, 49,50\% são do distrito de Coimbra, e 50,50\% de outro distrito fora de Coimbra.

Tabela 1

Distribuições absolutas e percentuais das variáveis sociodemográficas ( $N=188)$

\begin{tabular}{lcc}
\hline & $\mathrm{n}^{\mathbf{0}}$ & $\%$ \\
\hline Sexo: & 28 & 14,90 \\
Masculino & 160 & 85,10 \\
Feminino & & \\
\hline Residência antes do periodo de aulas: & 93 & 49,50 \\
Distrito de Coimbra & 95 & 50,50 \\
Fora do distrito de Coimbra
\end{tabular}

\section{METODOLOGIA E INSTRUMENTOS UTILIZADOS}

\section{Tipo de estudo}

Este estudo é de tipo prescritivo, seguindo um desenho pré-experimental, com grupo experimental único e avaliação pré e pós intervenção.

\section{Instrumento}

Como instrumento de colheita de dados recorreu-se ao QuALiSMental (Loureiro, 2015) na versão para a ansiedade social.

A primeira parte do QuALiSMental inclui as instruções de preenchimento e questões de caraterização sociodemográfica (sexo, idade e habilitações literárias dos pais).

A segunda parte é constituída por diferentes seções relativas às componentes em análise. Previamente é apresentada uma vinheta relatando um caso de ansiedade social de uma jovem chamada Rita, de acordo com os critérios de diagnóstico da DSM-5 (American Psychiatric Association, 2014). 0 conteúdo da vinheta é o seguinte:

"A Rita tem 18 anos e entrou no ensino superior em outubro do último ano, tendo mudado de cidade para frequentar o curso. Desde que mudou para a nova instituição, tornou-se ainda mais tímida do que habitualmente e fez apenas uma amiga. Ela gostaria de fazer mais amigos, mas tem medo de fazer ou dizer algo embaraçoso quando está com outras pessoas. Embora o desempenho escolar da Rita seja razoável, raramente participa nas aulas e fica muito nervosa, corada, a tremer e sente que pode vomitar se tiver de responder a alguma pergunta ou falar na frente da turma. Com a sua família, a Rita é muito conversadora, mas com os colegas da casa onde vive tal não aconte- 
ce, preferindo ficar calada quando recebem alguém que não conhece bem. À exceção dos telefonemas da família, nunca atende 0 telefone e recusa-se a participar em eventos sociais. Ela sabe que os seus medos são exagerados, mas não consegue controlá-los e isso realmente perturba-a."

\section{Procedimentos}

0 QuALiSMental foi administrado em ambos os momentos em sala de formação, pré e pós intervenção sob supervisão dos formadores. Previamente à administração do QuALiSMental, foi solicitada autorização à Presidente da Direção da instituição para a implementação do programa e ao Presidente do Conselho Pedagógico. 0 projeto foi submetido à Comissão de Ética da Unidade de Investigação em Ciências da Saúde: Enfermagem (UICISA: E) da Escola Superior de Enfermagem de Coimbra (N..:- P603-06/2019), tendo obtido parecer positivo.

A intervenção teve a duração de 9 horas, sendo constituída por 3 sessões de duas horas e uma sessão de 3 horas, tendo decorrido em setembro de 2018.

\section{Tratamento estatístico}

0 tratamento estatístico foi realizado com recurso ao software IBM-SPSS 24.0. Foram calculadas as estatísticas resumo adequadas (ex. média, desvio padrão) e as frequências absolutas e percentuais, antes e depois da formação.

Em termos de testes estatísticos, recorreu-se ao teste de McNemar, teste de Bowker e teste $\mathrm{t}$ para grupos emparelhados. No caso do teste de Bowker, e quando as diferenças observadas são estatisticamente significativas $(p<0,05)$, calcularam-se os procedimentos post hoc (à posteriori) com correção de Bonferroni.

Como medidas de tamanho de efeito foram calculadas, de acordo com a ordem dos testes referido anteriormente, as seguintes "g" de Cohen para o teste de McNemar (2x2); e para 0 teste de Bowker (LXC) e "d" de Cohen para o teste t (Cohen, 1988).

\section{RESULTADOS ALCANZADOS}

Ao nível da primeira componente em análise (reconhecimento da ansiedade social), como podemos observar da Tabela 1, após a formação, aumenta a utilização do rótulo "depressão" $(p=0,001$; $g=0,37)$, "doença mental" ( $p=0,001 ; g=0,39)$, "stresse" ( $p=0,052 ; g=0,10)$, e "ansiedade" ( $p=0,002$; $g=0,21$ ). No rótulo "esgotamento nervoso" as mudanças não têm significado estatístico, assim como no rótulo "problemas psicológicos/mentais/emocionais". É de salientar a descida na utilização do rótulo "crise da idade" ( $p=0,033 ; g=0,32)$.

Tabela 2

Distribuições percentuais dos rótulos assinalados antes de depois da intervenção, incluindo teste de McNemar e medida de tamanho de efeito $(N=188)$

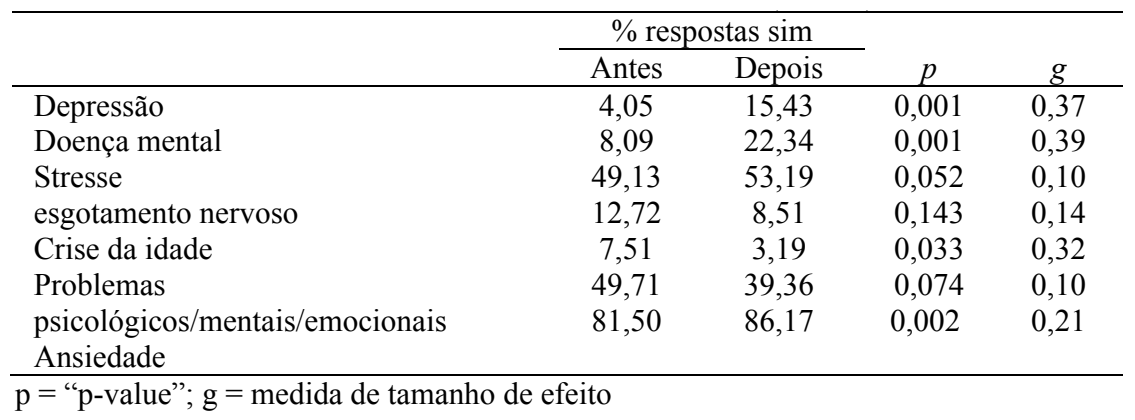


Quando questionados acerca da intenção de pedir ajuda profissional numa situação semelhante (Tabela 3), observa-se um incremento na intenção de pedido de ajuda $\left(c^{2}=41,279 ; p<0,001\right)$, sendo que no fim da intervenção, 16,49\% manifestam indecisão.

Tabela 3

Distribuições percentuais das respostas à questão "se estivesses a viver um problema semelhante procurarias ajuda?", antes e depois da intervenção. Inclui resultados do teste de Bowker (N=188).

\begin{tabular}{ccc|ccc|c}
\hline & Antes & \multicolumn{3}{|c}{ Depois } & \multirow{2}{*}{$\chi^{2}$} \\
\cline { 2 - 5 } Sim & Não & Não sei & Sim & Não & Não sei & $41,279 * * *$ \\
\hline ****
\end{tabular}

$\mathrm{p}<0,001$

Em termos de confiança para prestar ajuda (Tabela 4), no fim da intervenção observa-se um aumento com significado estatístico na confiança em prestar ajuda ( $t=-6,029 ; p<0,001 ; d=0,42)$ que é indicador de que o programa é efetivo em termos de contribuir para o incremento da literacia em saúde mental.

Tabela 4

Estatísticas resumo da confiança para prestar ajuda, antes e depois da intervenção ( $N=188)$.

\begin{tabular}{|c|c|c|c|c|c|c|}
\hline & \multicolumn{2}{|c|}{ Antes } & \multicolumn{2}{|c|}{ depois } & \multirow[b]{2}{*}{$t$} & \multirow[b]{2}{*}{$d$} \\
\hline & Média & DP & Média & DP & & \\
\hline $\begin{array}{l}\text { Confiança para ajudar } \\
{ }^{* * *} \mathrm{p}<0,001\end{array}$ & 3,29 & 0,89 & 3,54 & 0,73 & $-6,029^{* * *}$ & 0,42 \\
\hline
\end{tabular}

\section{DISCUSSÃO}

É de salientar ao iniciar a discussão algumas limitações, nomeadamente a utilização de um desenho que usa apenas um grupo experimental. Ainda que exista avaliação pré e pós intervenção, a inexistência de grupo de controle não possibilita a comparação dos resultados do programa com outro tipo de intervenção, assim como a ausência de follow-up não nos permite verificar como os estudantes que participaram no programa se apresentam passados alguns meses ao nível do seu conhecimento em termos de primeira ajuda, ou inclusive, se utilizaram os conhecimentos em seu proveito e daqueles que os rodeiam.

No que respeita à componente de reconhecimento da ansiedade social, após a intervenção, observa-se uma melhoria com significado estatístico em muitos rótulos, ainda que alguns não casos esses rótulos não se coadunem o problema descrito. Ainda que não seja objetivo do programa ensinar em nenhum momento a fazer diagnósticos em saúde mental, mas problematizar a situação descrita, é positivo verificar que aumenta a utilização dos rótulos "doença mental", "stresse" e "ansiedade".

Estes resultados estão em consonância com os observados noutros estudos realizados no contexto Português e que reportam a efetividade do programa em termos de reconhecimento dos problemas de saúde mental (Loureiro \& Sousa, 2019; Loureiro \& Costa, 2019; Loureiro \& Freitas, 2020a; Cunha, 2019; Cunha Loureiro, Rosa \& Costa, 2020), ou mesmo no contexto internacional, ainda que os problemas de problemas avaliados possam ser distintos (Bond et al., 2015; Burns et al., 2017; ; Morgan, Ross \& Reavley, 2018).

No que respeita à intenção de procura de ajuda numa situação semelhante, os resultados mostram que o programa é efetivo na manifestação desta intenção, tal como acontece na globalidade 
dos estudos (Bond et al., 2015; Burns et al., 2017; Loureiro \& Sousa, 2019; Loureiro \& Costa, 2019; Loureiro \& Freitas, 2020a; Cunha, 2019; Cunha Loureiro, Rosa \& Costa, 2020).

A principal mudança dá-se sobretudo daqueles que estão inicialmente relutantes ("não sei") e que são $35,64 \%$, que passam no fim da intervenção a ser apenas 16,49\%. Este resultado vem também ao encontro dos resultados dos estudos já referidos (Bond et al., 2015; Burns et al., 2017; Yee, 2018; ; Morgan, Ross \& Reavley, 2018; Loureiro \& Sousa, 2019; Loureiro \& Costa, 2019; Loureiro \& Freitas, 2020b; Cunha, 2019; Cunha Loureiro, Rosa \& Costa, 2020).

Por último, ao nível da confiança para prestar ajuda e apoio, os resultados indicam que o programa tem um efeito estatisticamente significativo, associado a um tamanho de efeito moderado, tal como 0 reportado noutros estudos (Bond et al., 2015; Burns et al., 2017; Loureiro \& Sousa, 2019; Loureiro \& Costa, 2019; Loureiro \& Freitas, 2020b; Cunha, 2019; Cunha, Loureiro, Rosa \& Costa, 2020). Como o objetivo do programa é aumentar a literacia em saúde mental, a tradução do resultado em termos de confiança para usar o conhecimento em proveito próprio ou de alguém que precise, é um indicar ótimo dos resultados deste programa.

A confiança manifestada pelos estudantes de Enfermagem que participaram no estudo é fundamental para que estes atuem nas situações do quotidiano associadas a saúde mental dos seus colegas. Naturalmente que o ensino do plano de ação que permite guiar os estudantes neste trabalho é fundamental para a sua ação, e o seu domínio reforça esta confiança para agir.

\section{CONCLUSÕES}

o programa educativo de primeira ajuda em saúde mental revela ser uma ferramenta efetiva como medida potenciadora da literacia em saúde mental dos estudantes universitários, especificamente no que respeita à ansiedade social.

0 programa poderá ser entendido como uma mais valia que permite aos estudantes agirem no seu quotidiano, nomeadamente ao nível da relação interpares, uma vez que este podem funcionar como impulsionadores da procura de ajuda em saúde mental.

Ainda que de forma indireta este programa pode ser entendido como uma medida que permite combater 0 estigma associado às doenças e doentes mentais, contribuindo para a visão de uma sociedade mais integradora, humana e tolerante, diminuindo a discriminação social que é ainda hoje visível no quotidiano.

\section{REFERENCIAS BIBLIOGRÁFICAS}

- Associação Americana de Psiquiatria. (2014). DSM-5: Manual de diagnóstico e estatística das perturbações (5aㅡ ed.). Porto Alegre, Brasil: Artmed Editora.

- Bond, K. S., Jorm, A. F., Kitchener, B. A., \& Reavley, N. J. (2015). Mental health first aid training for Australian medical and nursing students: an evaluation study. BMC Psychology, 3. http://doi: 10.1186/ s40359-015-0069-0

- Burns, S., Crawford, G., Hallett, J., Hunt, K., Chih, H., \& Tilley, P. (2017). What's wrong with John? A randomised controlled trial of Mental Health First Aid (MHFA) training with nursing students. BMC Psychiatry,17(1), 111. https://doi: 10.1186/s12888-017-1278-2

- Cohen, J. (1988). Statistical power analysis for the behavioral sciences. New Jersey, NJ: Lawrence Erlbaum Associates.

- Cunha, S. R. (2019). Efetividade do programa de primeiros socorros em saúde mental em estudantes do 1. ano de Enfermagem [Dissertação de mestrado, Escola Superior de Enfermagem de Coimbra]. Repositório Científico da Escola Superior de Enfermagem de Coimbra. http://web.esenfc.pt/?url=H4DEplcb 
- Cunha, S., Loureiro, L., Rosa, A., \& Costa, L. (2020). Efetividade do programa de primeiros socorros em saúde mental na literacia em saúde mental acerca da ansiedade social. Revista de Investigação em Enfermagem. (33). 35-44.

- Jorm, A. (2019). The concept of mental health literacy. In 0. Bauer, U. Levin-Zamir, D. P. Pinheiro \& K. Sorensen (Eds.), International handbook of health literacy: Research, practice and policy across the life-span (pp. 53-66). Policy Press.

- Jorm, A. F., Korten, A. E., Jacomb, P. A., Christensen, H., Rodgers, B., \& Pollitt, P. (1997). "Mental health literacy": A survey of the public $s$ ability to recognize mental disorders and their beliefs about the effectiveness of treatment. Medical Journal of Australia, 166(4), 182-186. https://doi.org/10.5694/j.1326-5377.1997.tb140071.x

- Loureiro, L. (2014). Primeira ajuda em saúde mental. Escola Superior de Enfermagem de Coimbra.

- Loureiro, L. (2015). Questionário de Avaliação da Literacia em Saúde Mental - QuALiSMental: Estudo das propriedades psicométricas. Revista de Enfermagem Referência, 4(4), 79-88. http://doi.org/10.12707/RIV14031

- Loureiro, L. M. \& Freitas, P. M. (2020a). Effectiveness of the mental health first aid program in undergraduate nursing students. Revista de Enfermagem Referência, 5(1), e19078. http://doi:10.12707/RIV19078

- Loureiro, L. M. J. (2020). Avaliação de impacto do programa de Primeira Ajuda em Saúde Mental na literacia em saúde mental dos estudantes do 1.ำ ano do curso de licenciatura em Enfermagem [Relatório de pós-doutoramento não publicado]. Universidade do Porto.

- Loureiro, L. M., Rosa, A., Frajuca, M., Cunha, S., Correia, S., Morgado, T., \& Costa, L. (2020). Primeira Ajuda em Saúde Mental: Contributo do programa para o incremento da literacia em saúde mental dos estudantes do ensino superior. Revista Portuguesa De Investigação Comportamental E Social, 6(2), 24-38. https://doi.org/10.31211/rpics.2020.6.2.184

- Loureiro, L., \& Costa, L. (2019). Avaliação do programa de primeiros socorros em saúde mental em estudantes de licenciatura em enfermagem. Revista de Enfermagem Referência, 4(20), 9-18. http://doi:10.12707/RIV18087

- Loureiro, L., \& Sousa, C. (2019). Programa de primeiros socorros em saúde mental: Estudo piloto. Revista Portuguesa de Investigação Comportamental e Social, 5(1), 72-86. http://doi:10.31211/rpics.2019.5.1.108

- Loureiro, Luís, \& Freitas, Paula. (2020b). Literacia em saúde mental dos jovens estudantes de enfermagem na integração ao ensino superior. Revista Portuguesa de Enfermagem de Saúde Mental, (24), 34-42. https://dx.doi.org/10.19131/rpesm.0279

- Morgan, A. J., Ross, A., \& Reavley, N. J. (2018). Systematic review and meta-analysis of mental health first aid training: Effects on knowledge, stigma, and helping behaviour. PLOS ONE, 13(5), e0197102. http://doi.org/10.1371/journal.pone.0197102 
